\title{
A New Pair of Pentaketide Diastereoisomers from Aspergillus melleus YIM PHI001
}

\section{Qiang Wang $^{\oplus 1}$, Xue-Qiong Yang ${ }^{\oplus 2}$, Cui-Ping Miao ${ }^{\oplus 1}$, Li-Hua Xu ${ }^{\oplus 1}$, Zhong-Tao Ding $^{\oplus 2}$, Ya-Bin Yang ${ }^{\oplus 2^{*}}$ and Li-Xing Zhao ${ }^{\oplus *}$}

\author{
${ }^{1}$ Yunnan Institute of Microbiology, School of Life Science, Yunnan University, Kunming 650091, \\ People's Republic of China \\ ${ }^{2}$ Key Laboratory of Medicinal Chemistry for Natural Resource, Ministry of Education, School of \\ Chemical Science and Technology, Yunnan University, Kunming 650091, People's Republic of China
}

(Received April 3, 2017; Revised July 13, 2017; Accepted August 23, 2017)

\begin{abstract}
Two new aspinotriol derivatives (1-2) determined as melleusin A (1), B (2) and other seven known compounds were isolated from a soil-borne fungus Aspergillus melleus. The configurations of $\mathbf{1}$, and $\mathbf{2}$ were determined by their analogues, aspilactonol B, C isolated previous in Aspergillus. Mellein (8) showed broad antibiotic activity against the test pathogens. Aspinonene, aspinotriols A and B can be used as the marker molecule in chemotaxonomy of Aspergillus.
\end{abstract}

Keywords: Aspergillus melleus; aspinotriol derivatives; diastereoisomer; spectroscopic analyses; antibiotic. (C) 2018 ACG Publications. All rights reserved.

\section{Introduction}

The genus Aspergillus is rich in species and serves as a reservoir of bioactive secondary metabolites [1]. The potential of finding even further new bioactive drug candidates in Aspergillus is evident, despite the fact that many secondary metabolites have already been structure elucidated and chemotaxonomic studies have shown that many new secondary metabolites have yet to be characterized [2]. Aspyrone, a weak broad spectrum antibiotic produced by the Aspergillus melleus, is a polyketide derived from five $\mathrm{C}_{2}$ units [3]. During ongoing search for new natural products from microbes living in untapped niches, Aspergillus melleus YIM PHI001 isolated from an India soil sample was screened and two new aspinotriol derivatives (1-2) were obtained from its culture extract (Figure 1). Herein, we present the isolation, structure elucidation and bioactivities of the two new compounds.

\footnotetext{
*Corresponding authors: E-Mail: zlx70@163.com (L. X. Zhao); ybyang@ynu.edu.cn (Y. B. Yang)
} 


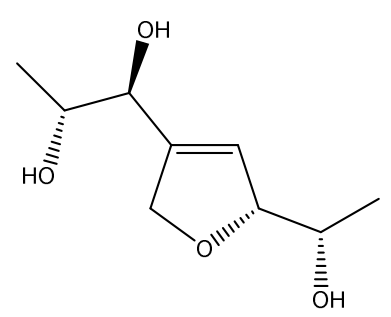

1<smiles>C[C@H](O)[C@H](O)C1=C[C@@H]([C@@H](C)O)CC1</smiles>

2<smiles>CC1OC1[C@@](O)(/C=C/[C@H](C)O)CO</smiles>

4<smiles>C[C@H](O)/C=C/C(=C\[C@H](C)O)CO</smiles>

5<smiles>C[C@H](O)/C=C/C(=C/[C@@H](C)O)CO</smiles>

6

Figure 1. Structures of compounds 1 and 2, and 4-6

\section{Materials and Methods}

\subsection{General Experimental Procedures}

Silica gel (200-300 mesh; Qingdao Marine Chemical Group Co.), MCI (The Kaiteki Co.), ODS (Beijing Greenherbs and Technology and Development Co.) and Sephadex LH-20 (GE Healthcare Co.) were used for CC. 1D and 2D NMR spectra were obtained on a Bruker 400, 500, 600 $\mathrm{MHz}$ NMR instrument (Bruker). MS spectra were recorded with an Agilent G3250AA (Agilent) and AutoSpec Premier P776 spectrometer (Waters).

\subsection{Fungal Material}

The fungus strain was isolated from the soil in New Delhi, India. The species was identified as Aspergillus melleus (YIM PHI001) based on morphological, genetic (ITS) and pathogenicity analyses. A voucher specimen was deposited at the Yunnan Institute of Microbiology, Kunming, P.R. China.

\subsection{Fermentation}

Aspergillus melleus YIM PHI001 was maintained on the PDA medium. Small agar plugs (approximately $5 \mathrm{~mm} \times 5 \mathrm{~mm}$ ) of the fungus were cultured in $0.5 \mathrm{~L}$ Erlenmeyer flasks containing 100 $\mathrm{mL}$ of modified martin medium at $130 \mathrm{rpm}$ and $28^{\circ} \mathrm{C}$ for 3 days as a seed culture. Then a $50 \mathrm{~mL}$ seed culture was transferred into a $1 \mathrm{~L}$ Erlenmeyer flask containing $200 \mathrm{~mL}$ of potato dextrose broth (PDB) and incubated at $130 \mathrm{rpm}$ and $28^{\circ} \mathrm{C}$ for 15 days. A total $55 \mathrm{~L}$ fermentation broth was collected.

\subsection{Extraction and Isolation of Compounds}

The production broth $(55 \mathrm{~L})$ was centrifuged to separate the mycelia from the suspension. The broth was exhaustively extracted with EtOAc, yielding $16.8 \mathrm{~g}$ of extract. The mycelia were extracted three times with methanol with ultrasonic processing for $30 \mathrm{~min}$ each time. The methanol extract was removed under vacuum, and the resulting aqueous layer was extracted three times with an equal 
volume of EtOAc to yield a crude extract $(30.5 \mathrm{~g})$. The extracts of the fermentation broth and the mycelia were separated after TLC and HPLC analyses. The organic extract of mycelia was first subjected to $\mathrm{CC}$ over silica gel eluted with stepwise $\mathrm{CHCl}_{3} / \mathrm{MeOH}$ gradient $(100: 0,50: 1,20: 1$, and $5: 1, \mathrm{v} / \mathrm{v})$ to yield Fractions 1-7. Fr. 1 was fractioned by column chromatography on silica gel eluted with petroleum ether $/ \mathrm{CHCl}_{3}(5: 1,1: 1)$ to yield Fr.1.1- Fr.1.3. Fr. 1.3 was eluted upon Sephadex LH-20 $\left(\mathrm{CHCl}_{3} / \mathrm{MeOH}=1: 1\right)$ to obtain $8(8 \mathrm{mg})$. Fr. 2 was fractioned by column chromatography on silica gel eluted with petroleum ether/ $\mathrm{CHCl}_{3}(1: 1)$ to yield Fr. 2.1-Fr. 2.3. Fr. 2.2 was fractioned by ODS eluted with acetone $/ \mathrm{H}_{2} \mathrm{O}(20 \%$ to $100 \%)$ to obtain $7(11 \mathrm{mg})$. Fr. 2.3 was fractioned by ODS eluted with acetonitrile $/ \mathrm{H}_{2} \mathrm{O}(60 \%$ to $100 \%)$ to obtain $9(15 \mathrm{mg})$. Fr. 5 was fractioned by column chromatography on silica gel eluted with petroleum ether/acetone (6:4) to obtain Fr.5.1-Fr.5.3. The sub-fraction Fr. 5.3 was eluted upon Sephadex LH-20 $\left(\mathrm{CHCl}_{3} / \mathrm{MeOH}=1: 1\right)$ and further purified by ODS eluted with acetonitrile $/ \mathrm{H}_{2} \mathrm{O}(10 \%-30 \%, \mathrm{v} / \mathrm{v})$ to afford compounds $\mathbf{1}, \mathbf{2}(8 \mathrm{mg}), \mathbf{4}(6 \mathrm{mg}), \mathbf{5}(7 \mathrm{mg}), \mathbf{6}(11 \mathrm{mg})$. Fr. 6 was fractioned by column chromatography on silica gel eluted with $\mathrm{CHCl}_{3} / \mathrm{MeOH}$ gradient to yield Fr. 6.1-Fr. 6.2. Fr. 6.2 was eluted upon Sephadex $\mathrm{LH}-20\left(\mathrm{CHCl}_{3} / \mathrm{MeOH}=1: 1\right)$ to obtain 3 (6 mg).

\subsection{Antimicrobial Activity Assays}

Compounds 1-9 were evaluated for antimicrobial activities. The assays were performed in 96well sterilized microplates using a microdilution method [4]. Kanamycin and nystatin (Taicheng Pharmaceutical Co., purity $>99 \%$ ) were introduced as the positive controls. Each concentration was tested in triplicate. Microbial growth was observed with a CX21BIM-set 5 microscope (Olymps Corp). MICs were determined as the lowest concentrations that produced complete growth inhibition of the microorganisms tested.

\section{Results and Discussion}

HR-ESIMS analysis of compounds $\mathbf{1}$, and $\mathbf{2}$ as stereoisomerism mixture (2 for minor constituent) revealed quasi-molecular ion peak at 211.0936[M+Na $]^{+}$, calcd. 211.0946, respectively. The ${ }^{1} \mathrm{H}$ and ${ }^{13} \mathrm{C}$ NMR spectra data (Table 1), including DEPT, clearly showed two groups of signals about 1 and 2 including two methyls, one methylene, five methines (one for olefinic bond), one olefinic quaternary carbon, which showed the skeleton of pentaketides. Aspilactonol B, C [5], aspinonene (4), aspinotriols A (5) and B (6) [6] sharing the similar structure with $\mathbf{1}$ and $\mathbf{2}$ were also produced by Aspergillus. The signals of ${ }^{1} \mathrm{H}-\mathrm{NMR}$ and ${ }^{13} \mathrm{C}-\mathrm{NMR}$ were assigned according to the correlations in the 2D NMR spectra and the chemical shift values. The fragments of $\mathrm{H}-1$ to $\mathrm{H}-4$ and $\mathrm{H}-$ 7 to H-9 was established on the basis of the COSY correlations, and the HMBC correlations from H-1 to $\mathrm{C}-2$, 3, from $\mathrm{H}-9$ to $\mathrm{C}-7$, 8, from $\mathrm{H}-6$ to $\mathrm{C}-4$, 5, from $\mathrm{H}-4$ to $\mathrm{C}-3$, from $\mathrm{H}-7$ to $\mathrm{C}-6$ confirmed the common skeleton of compounds 1, 2 (Figure 2). The key connection of C-3 and C-6 was confirmed by the NMR data at C-3, and C-6 (Table1). The structures of $\mathbf{1 , 2}$ and the known compounds, aspilactonol $\mathrm{B}, \mathrm{C}$ were almost the same, except for a methylene in 1, 2 changed to a carbonyl in aspilactonol $\mathrm{B}, \mathrm{C}$. The stereochemistry of these new compounds in C-2, C-3, C-7, C-8 was determined the same as aspilactonol B, C for $S, R, S, R$ in $\mathbf{1}$ and $S, R, R, S$ in $\mathbf{2}$ by comparing the NMR data and biogenesis (Figure 1). So these two stereomers were determined as melleusin A (1), B (2). Metabolites 1, 2 were derived from a common biosynthetic precursor, $\mathbf{A}-\mathbf{3}$, which originates from the intermediate $\mathbf{A}$, the ultimate product of polyketide synthesis (PKS), by post-PKS modifications (Figure 3). Reduction of the aldehyde in A-3 into the primary alcohol give A-4, and formation of $\mathbf{1}, 2$ by nucleophilic attack of the primary alcohol on either site of the carbons in one of the two epoxide groups and hydration in the rest epoxide group, and the similar biosynthetic conversion was also reported in aspilactonol B, C [5]. 


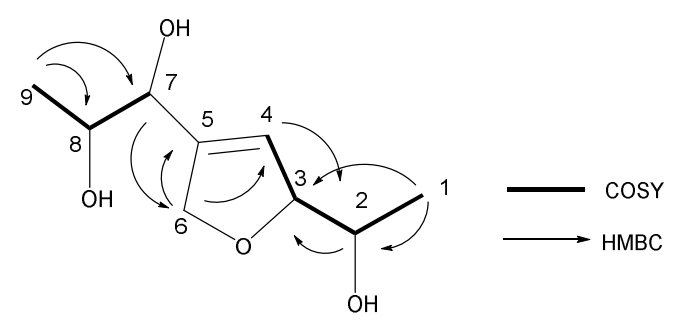

Figure 2. The key COSY and HMBC correlations of compounds $\mathbf{1}$ and 2.

Table 1. ${ }^{1} \mathrm{H}$ and ${ }^{13} \mathrm{C}$ NMR Data of $\mathbf{1 , 2}$ in $\operatorname{MeOD}(\delta$ in ppm, $J$ in $\mathrm{Hz})$

\begin{tabular}{ccccc}
\hline \multirow{2}{*}{ Position } & \multicolumn{1}{c}{$\mathbf{1}$} & \multicolumn{2}{c}{$\mathbf{2}$} \\
\cline { 2 - 5 } & $\delta_{\mathrm{H}}$ & $\delta_{\mathrm{C}}$ & $\delta_{\mathrm{H}}$ & $\delta_{\mathrm{C}}$ \\
\hline 1 & $1.16(\mathrm{~d}, 5.0)$ & 17.3 & $1.22(\mathrm{~d}, 6.0)$ & 17.6 \\
2 & $3.74(\mathrm{~m})$ & 69.2 & $3.74(\mathrm{~m})$ & 69.3 \\
3 & $4.67(\mathrm{~m})$ & 90.5 & $4.67(\mathrm{~m})$ & 90.5 \\
4 & $5.80(\mathrm{~s})$ & 121.9 & $5.80(\mathrm{~s})$ & 121.9 \\
5 & & 143.1 & & 143.2 \\
6 & $4.64,4.73(\mathrm{~m})$ & 74.9 & $4.64,4.73(\mathrm{~m})$ & 74.9 \\
7 & $4.17(\mathrm{~d}, 5.5)$ & 72.6 & $4.17(\mathrm{~d}, 5.5)$ & 72.7 \\
8 & $3.74(\mathrm{~m})$ & 69.6 & $3.74(\mathrm{~m})$ & 69.7 \\
9 & $1.17(\mathrm{~d}, 6.0)$ & 17.4 & $1.21(\mathrm{~d}, 6.0)$ & 17.5 \\
\hline
\end{tabular}

$\mathrm{C}_{2}$-building blocks $\times 5$<smiles>C/C=C/C(C=O)/C=C/C(O)CC(=O)OC(=O)CC(O)/C=C/C1OC1/C=C/C</smiles><smiles>C/C=C\C(C=O)=C/C=C/C(=O)O</smiles>

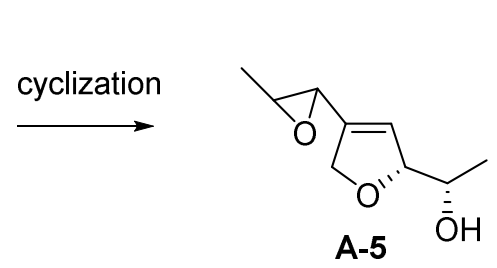<smiles>CC(O)C1=CC(C(C)O)OC1</smiles><smiles>CC(O)[C@H]1C=C([C@@H](O)C(C)O)OC1</smiles>

Figure 3. The possible biosynthetic pathway of new compounds 1-2.

Other known compounds were isolated and determined as chrysogeside D (3) [7], aspinonene (4) [6], aspinotriols A (5) and B (6) [6], 22E,24R-ergosta-7,22-diene-3 $\beta, 5 \alpha, 6 \beta$-triol (7) [8], mellein (8) [9], 4-hydroxymellein (9) [10].

The mixture of compound $\mathbf{1}$ and $\mathbf{2}$, and other isolated compounds (3-9) were evaluated for 
antimicrobial activity against the pathogenic bacteria and fungi, Escherichia coli, Bacillus subtilis, Candida albicans, Fusarium oxysporum, Fusarium solani. Compound $\mathbf{8}$ indicated the broad spectrum antibiotic activity against all five pathogens with MICs at $128-256 \mu \mathrm{g} / \mathrm{mL}$. As positive controls, kanamycin had antibacterial activity against $E$. coli, B. subtilis with MICs of $32 \mu \mathrm{g} / \mathrm{mL}$, nystatin had antifungal activity against $C$. albicans, $F$. oxysporum, and $F$. solani with MICs of 16,32 , and 16 $\mu \mathrm{g} / \mathrm{mL}$. Control of harmful microorganism in food, aquaculture, pharmaceuticals, agriculture, hospitals and recreation water pools are great global concern. Some infections maynot caused by single pathogen, but multi-pathogens, so the metabolites with broad antibiotic activity had significance on control of these multi-pathogens. In this research, we found mellein had antibacterial and antifungal activities, and it can be used as a biocontrol against disease caused by bacteria, and fungi. Previous works showed that aspinonene, aspinotriols $\mathrm{A}$ and $\mathrm{B}$ were mainly found in Aspergillus, so these compounds can be used as marker molecule in chemotaxonomy of Aspergillus.

\section{Acknowledgments}

This work was partly supported by the National Natural Science Foundation of China [grant number 81360480], [grant number 21562045], [grant number 81460536].

\section{Supporting Information}

Supporting Information accompanies this paper on http://www.acgpubs.org/RNP

\section{ORCID}

Qiang Wang : 0000-0003-4391-5422

Xue-Qiong Yang: 0000-0002-3259-5737

Cui-Ping Miao: 0000-0002-3084-8091

Li-Hua Xu: 0000-0002-7852-6286

Zhong-Tao Ding: 0000-0002-7860-060X

Ya-Bin Yang: 0000-0001-5509-4559

and Li-Xing Zhao: 0000-0002-1353-3717

\section{References}

[1] H. W. Zhang, Y. F. Tang, C. F. Ruan and X. L. Bai (2016). Bioactive secondary metabolites from the endophytic Aspergillus genus, Rec. Nat. Prod. 10, 1-16.

[2] J. C. Frisvad and T.O. Larsen (2015). Chemodiversity in the genus Aspergillus, Appl. Microbiol. Biotechnol. 99, 7859-7877.

[3] A. M. Hill, A. Jacobs and J. Staunton (1995). Investigation of the stereochemistry of the tri- and tetraketide hydroxyacyl intermediates in the biosynthesis of the polyketide aspyrone in Aspergillus malleus using deuterium labelling and deuterium NMR spectroscopy, J. Chem. Soc. Chem. Commun. 8, 859-860.

[4] H. Zhou, L. X. Zhao, W. Li, Y. B. Yang, L. H. Xu, and Z. T. Ding (2015). Anti-Mycobacterium tuberculosis active metabolites from an endophytic Streptomyces sp. YIM65484, Rec. Nat. Prod. 9, 196-200.

[5] X. W. Chen, C. W. Li, C. B. Cui, W. Hua, T.J. Zhu and Q. Q. Gu (2014) Nine new and five known polyketides derived from a deep sea-sourced Aspergillus sp. 16-02-1, Mar. Drugs 12, 3116-3137.

[6] K. Kito, R. Ookura, S. Yoshida, M. Namikoshi, T. Ooi and T. Kusumi (2007). Pentaketides relating to aspinonene and dihydroaspyrone from a marine-derived fungus, Aspergillus ostianus, J. Nat. Prod. 70, 2022-2025.

[7] Y. Y. Li, P. Liu, Y. Tang, H. M. Li, Y. L. Tang, X. H. Liang and Y. J. Tang (2013). Novel cerebrosides isolated from the fermentation mycelia of Tuber indicum, Helv. Chim. Acta 96, 702-709.

[8] W. Li, X. Q. Yang, Y. B. Yang, S. H. Qin, Q. L. Li, L. X. Zhao and Z. T. Ding (2015). A new natural nucleotide and other antibacterial metabolites from an endophytic Nocardia sp., Nat. Prod. Res. 29, 132136. 
[9] N. Kamal, C. V. Viegelmann, C. J. Clements and R. A. Edrada-Ebel. Metabolomics-guided isolation of antitrypanosomal metabolites from the endophytic fungus Lasiodiplodia theobromae, Planta Med. DOI: $10.1055 / \mathrm{s}-0042-118601$.

[10] C. H. Lu, S. S. Liu, J. Y. Wang, M. Z. Wang and Y. M. Shen (2014). Characterization of eight new secondary metabolites from the mutant strain G-444 of Tubercularia sp. TF5, Helv. Chim. Acta, 97, 334344.

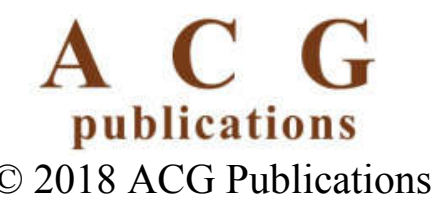

